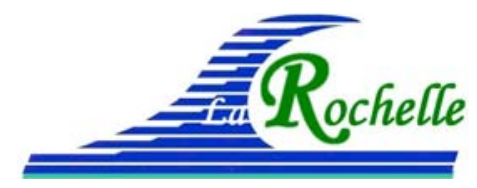

XVèmes Journées Nationales Génie Côtier - Génie Civil

La Rochelle, 29 au 31 mai 2018

DOI:10.5150/jngcgc.2018.061 C Editions Paralia CFL

disponible en ligne - http://www.paralia.fr - available online

\title{
Evaluation de la première fréquence propre d'un modèle réduit d'éolienne offshore fondé sur monopieu
}

\author{
Laura KERNER ${ }^{1}$, Jean-Claude DUPLA ${ }^{2}$, Gwendal CUMUNEL ${ }^{2}$, \\ Jean-Michel PEREIRA ${ }^{2}$, Jean CANOU ${ }^{2}$, Pierre ARGOUL ${ }^{3}$
}

1. CEREMA, DTITM, 110 rue de Paris, 77171 Sourdun, France. laura.kerner@cerema.fr

2. Université Paris-Est, Laboratoire Navier (UMR 8205), CNRS, ENPC, IFSTTAR, 6 8 avenue Blaise Pascal, 77455 Marne-la-Vallée Cedex 2, France.

3. IFSTTAR, Laboratoire MAST-EMGCU, 14 - 20 boulevard Newton, 77455 Marnela-Vallée Cedex 2, France.

\section{Résumé :}

Les éoliennes en mer, en pleine expansion en Europe, sont des structures élancées avec une première fréquence propre fortement dépendante de l'interaction sol-structure. Ce type de structure est soumis à un chargement latéral cyclique et dynamique induit par le vent, la houle et les courants mais aussi la rotation des pales. Les fréquences de ces sollicitations sont très proches de la première fréquence propre de l'éolienne. De fait, les éoliennes offshore typiquement installées et dénommées "soft-stiff" ont leur première fréquence propre qui s'insère dans un intervalle restreint compris entre la fréquence du rotor et celle des pales.

Pour éviter tout phénomène de résonnance, il est donc nécessaire de pouvoir évaluer précisément cette grandeur. Or, les éoliennes sont actuellement dimensionnées à partir des guides relatifs aux plateformes pétrolières offshores dont les fondations sont des pieux flexibles ayant un fort élancement. Au contraire, les monopieux, qui représentent $80 \%$ des fondations mises en place pour les éoliennes en mer, ont un diamètre large, un élancement faible (L/D compris entre 4 et 6 ) et un comportement rigide.

Dans ce contexte, une étude comparative de différentes méthodes d'évaluation de la première fréquence propre d'une telle structure (de référence ou innovantes) est proposée en se basant sur un modèle réduit d'éolienne à l'échelle 1/60. Les résultats obtenus permettent à la fois de souligner les imperfections de la méthode de référence et de mettre en exergue les méthodes les plus simples et les plus efficaces.

Mots-clés : Eolienne offshore, Monopieu, Fréquence propre, Interaction sol-structure.

\section{Introduction}

Le développement des éoliennes en mer est exponentiel en Europe ces dernières années avec un objectif commun à atteindre de $20 \%$ d'énergies renouvelables dans le mix énergétique à l'horizon 2020. Une éolienne typique installée actuellement est soumise à un large panel de chargements cycliques dynamiques. De fait, une telle structure subit le 


\section{Thème 5 - Énergies et ressources marines}

vent, la houle et les courants dont les fréquences sont en moyenne inférieures à $0,2 \mathrm{~Hz}$. Parmi les fréquences d'excitation, la fréquence du rotor $(1 P)$ ainsi que la fréquence de passage des pales $(3 P)$ doivent être considérées. L'ensemble de ces fréquences est reporté dans la figure 1 pour trois éoliennes existantes ayant une capacité allant de 3 à 8 MW (Vestas V90, Siemens 6 MW, Vestas V164) et une éolienne de référence définie par le NREL de 5 MW (JONKMAN et al., 2009). Pour éviter tout phénomène de résonance, l'éolienne est dimensionnée afin d'avoir la première fréquence propre comprise entre les intervalles $1 P$ et $3 P$. Une telle structure est appelée "soft-stiff", c'està-dire avec un mât souple (soft) et une fondation rigide (stiff).

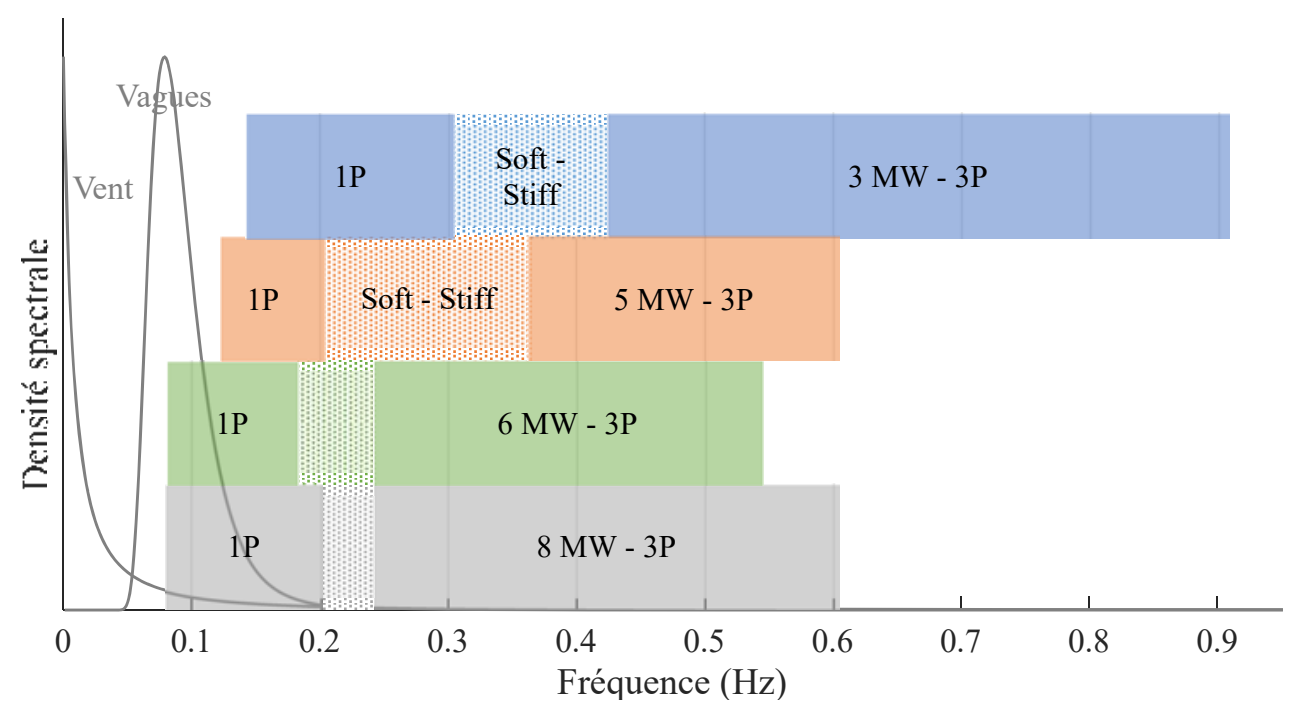

Figure 1. Ensemble des fréquences agissant sur les éoliennes Vestas V90 3 MW, NREL 5 MW, Siemens 6 MW et Vestas V164 8 MW.

En considérant les trois éoliennes existantes dont les fréquences d'excitation sont récapitulées sur la figure 1 , force est de constater que l'intervalle "soft-stiff" tend à diminuer avec l'augmentation de la capacité de l'éolienne offshore. Ainsi, il est primordial de pouvoir évaluer finement la première fréquence propre de cette structure. Dans la présente étude, différentes méthodes existantes pour l'évaluation de la première fréquence propre d'une éolienne offshore sont décrites. En se basant sur un modèle réduit à l'échelle 1/60 d'une éolienne en mer fondée sur monopieu, l'ensemble de ces méthodes sont comparées et discutées.

\section{Etat de l'art : méthode d'évaluation de la première fréquence propre d'une éolienne offshore}

Selon KALLEHAVE et al. (2015), l'interaction sol-structure est le facteur influençant le plus le comportement d'une éolienne en mer et sa première fréquence propre. La 


\section{XVèmes Journées Nationales Génie Côtier - Génie Civil \\ La Rochelle, 29 au 31 mai 2018}

méthode de référence ainsi que plusieurs méthodes existantes sont présentées ci-après. Ces méthodes se basent sur différentes représentations du monopieu et de l'interaction sol-structure comme illustré dans la figure 2 .

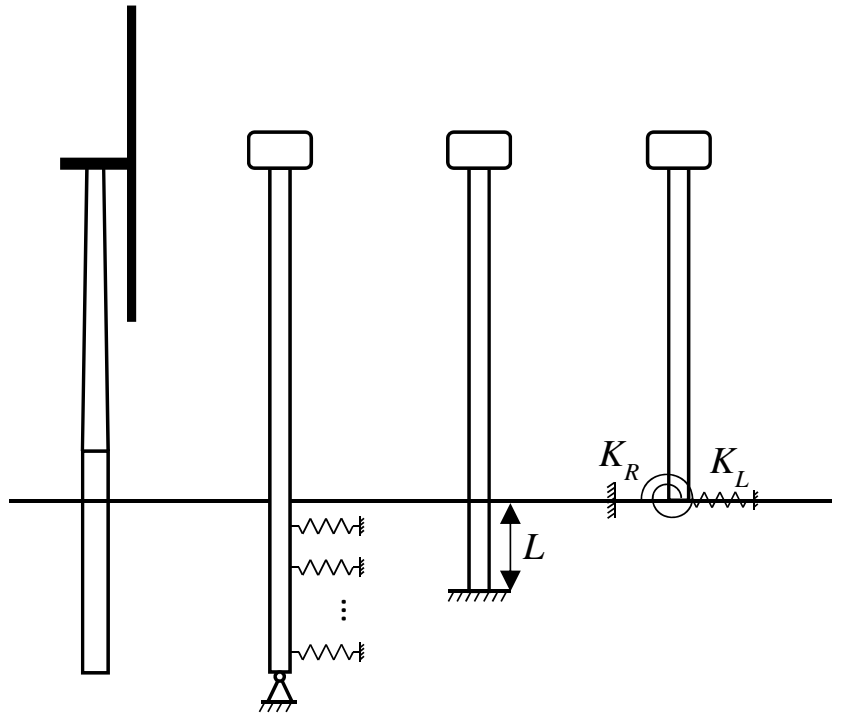
(a)
(b)
(c)
(d)

Figure 2. (a) Eolienne de référence ; (b) modèle de Winkler associé aux courbes p-y ;

(c) longueur d'encastrement équivalente;

(d) fondation remplacée par un ensemble de ressorts.

\subsection{Méthode de référence}

L'évaluation de la première fréquence propre d'une éolienne offshore se fait actuellement à l'aide des guides de référence (API, 2000 ; DNV, 2010). La structure est modélisée par une poutre d'Euler Bernoulli et l'interaction sol-structure est modélisée par un ensemble de ressorts découplés latéraux répartis le long de la fondation (modèle de Winkler) comme illustré sur la figure 2 (b). La raideur des ressorts est fonction de la distance entre les ressorts ainsi que du coefficient de réaction du sol $E_{p y}$, qui est évalué à partir des courbes $p-y$ établies pour les sables et les argiles. $E_{p y}$ correspond à la tangente à l'origine des courbes $p-y$ tel que :

$E_{p y}=\left(\frac{d p}{d y}\right)_{y=0}=k z$

avec $k$, le module de réaction initial $(\mathrm{Pa} / \mathrm{m})$ fonction de l'angle de frottement interne et/ou de l'indice de densité et $z$, la profondeur considérée.

L'application directe des courbes $p-y$ dans le cadre des éoliennes offshore est néanmoins discutable puisqu'elles ont été calibrées à partir d'essais in situ réalisées par MURCHISON \& O'NEIL (1984) pour deux pieux ayant un élancement $L / D$ de 34,4 , 


\section{Thème 5 - Énergies et ressources marines}

soit des pieux ayant un comportement flexible. En comparaison, un monopieu d'une éolienne à un élancement compris entre 4 et 6 , soit un comportement quasiment rigide. Par ailleurs, des mesures in situ sur trois éoliennes offshore du parc de Walney au Royaume-Uni, présentent une erreur moyenne oscillant entre 5 et $7 \%$ entre la première fréquence propre estimée et celle calculée. Il s'agit d'une sous-estimation de l'interaction sol-structure avec une fréquence propre réelle supérieure à la celle dimensionnée. Pour pallier ce problème, de nouvelles formulations des courbes $p-y$ prenant en compte notamment le diamètre de la structure et les caractéristiques du sol ont été développées.

\subsection{Méthode de référence modifiée}

Ainsi pour une éolienne offshore soumise à un chargement de type état limite de service (ELS), KALLEHAVE et al. (2012) ont développé une nouvelle expression pour le coefficient de réaction du sol $E_{p y}$ comme suit :

$E_{\text {py }}=k z_{\text {ref }}\left(\frac{z}{z_{\text {ref }}}\right)^{b}\left(\frac{D}{D_{\text {ref }}}\right)^{c}$

avec : $k$ le module de réaction initial $(\mathrm{Pa} / \mathrm{m}), D_{r e f}=0,61 \mathrm{~m}$ le diamètre de référence, $Z_{r e f}=2,5 \mathrm{~m}$ la profondeur de référence, et $b=0,6 ; c=0,5$ des paramètres adimensionnels. D'autres formulations ont été élaborées avec notamment les travaux de SØRENSEN et al. (2010) concernant l'expression du coefficient de réaction du sol pour une éolienne offshore soumise à un chargement ultime (ELU) et les recherches de THIEKEN et al. (2015) qui propose une définition de $E_{p y}$ prenant en compte à la fois l'ELS et l'ELU.

\subsection{Méthode de longueur encastrée équivalente}

La méthode existante la plus aisée consiste à remplacer l'ensemble sol/fondation par un pieu encastré d'une longueur $L$ donnée comme illustré sur la figure 2 (c). Selon KÜHN et al. (1998), cette longueur est comprise entre 3,3 et 3,7 fois le diamètre du pieu pour les éoliennes offshore fondées sur monopieu.

\subsection{Fondation modélisée par un ensemble de ressorts}

ADHIKARI \& BHATTACHARYA (2012) proposent une approche assez simple pour l'étude des fréquences propres d'une éolienne offshore en prenant en compte l'interaction sol-structure. La structure est modélisée par une poutre d'Euler Bernoulli avec une masse en tête. La poutre repose sur un ensemble de ressorts représentant la fondation et son interaction avec le sol comme illustré sur la figure 2 (d). A partir de ce modèle, une équation caractéristique permet ainsi de remonter aux fréquences propres de la structure. ADHIKARI \& BHATTACHARYA (2012) ont défini deux méthodes analytiques permettant d'évaluer la valeur de la raideur du ressort latéral $\left(K_{L}\right)$ et du ressort de torsion $\left(K_{R}\right)$. La première repose sur l'Eurocode 8 (2004) dont l'annexe C 


\section{XVèmes Journées Nationales Génie Côtier - Génie Civil \\ La Rochelle, 29 au 31 mai 2018}

définit l'expression de la raideur statique de pieux flexibles encastrés pour trois modèles de sol (correspondant à trois définitions différentes de l'évolution du module d'Young avec la profondeur). Une deuxième méthode analytique, développée par ADHIKARI \& BHATTACHARYA (2012), permet d'exprimer les raideurs des ressorts comme suit :

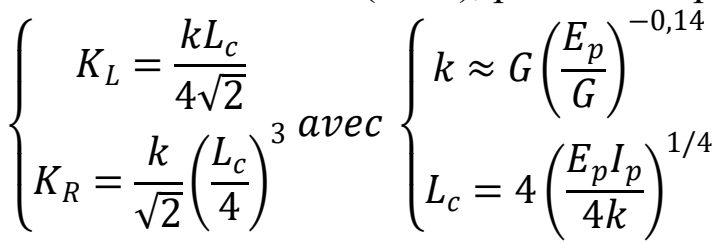

avec : $G$ le module de cisaillement du sol $(\mathrm{Pa}), I_{p}$ le oment quadratique du monopieu $\left(\mathrm{m}^{4}\right)$ et $E_{p}$ le module d'Young du monopieu $(\mathrm{Pa})$.

Les quatre méthodes présentées sont considérées dans la partie suivante et permettent de comparer les différentes méthodes existantes et de souligner leurs limites. La méthode de référence est notée $M 1$, la méthode de KALLEHAVE et al. (2012), M2, la méthode de longueur encastrée équivalente $M 3$ et la méthode D'ADHIKARI \& BHATTACHARYA (2012), M4.

\section{Evaluation de la première fréquence propre d'un modèle réduit : étude comparative}

Obtenir l'ensemble des dimensions d'une éolienne en mer réelle ainsi que ses fréquences propres est particulièrement difficile. Ainsi, cette étude se base sur l'éolienne de référence développée par le NREL (JONKMAN et al., 2009), dont l'ensemble des caractéristiques est défini. Un modèle réduit à l'échelle 1/60 de l'éolienne NREL $5 \mathrm{MW}$ a été mis en place afin d'obtenir des valeurs de fréquence propre de référence. Ces valeurs permettent ainsi de comparer les méthodes présentées dans la partie précédente.

\subsection{Modèle réduit à l'échelle $1 / 60$}

Le modèle réduit est constitué d'un mât et d'un monopieu en acier inoxydable. Le modèle est installé dans un massif de sable de Fontainebleau (NE34). Les caractéristiques principales du modèle réduit sont récapitulées sur la figure 3 . Le pieu a un comportement analogue à une éolienne réelle. En effet, avec un diamètre de $80 \mathrm{~mm}$ et une fiche de $600 \mathrm{~mm}$, le monopieu a un élancement de 7,5, proche de l'élancement typique d'une telle fondation. L'interaction sol-structure étant un des facteurs majeurs influençant la première fréquence propre d'une éolienne, une baudruche en néoprène est installée en tête du massif de sable. Cette baudruche permet d'appliquer une pression uniforme sur la surface du massif. Une contrainte verticale $\sigma_{V}^{\prime}$ allant de 0 à $200 \mathrm{kPa}$ a donc été appliquée sur le massif, ce qui permet de faire varier la rigidité du sol.

Des essais de vibration libre ont été effectués sur le modèle réduit afin d'évaluer la première fréquence propre pour diverses contraintes verticales. A l'aide d'un marteau 


\section{Thème 5 - Énergies et ressources marines}

d'impact (Brüel et Kjær type 8206), un choc bref est appliqué en tête du mât et la vibration libre de la structure est enregistrée à l'aide d'un accéléromètre collé sur le modèle réduit comme illustré sur la figure 3. La première fréquence propre est obtenue à l'aide de la méthode d'identification modale utilisant la transformée en ondelettes (LE \& ARGOUL, 2004) appliquée aux réponses après impact du modèle réduit. Les résultats obtenus sont présentés sur la figure 4 (courbe noire "exp").
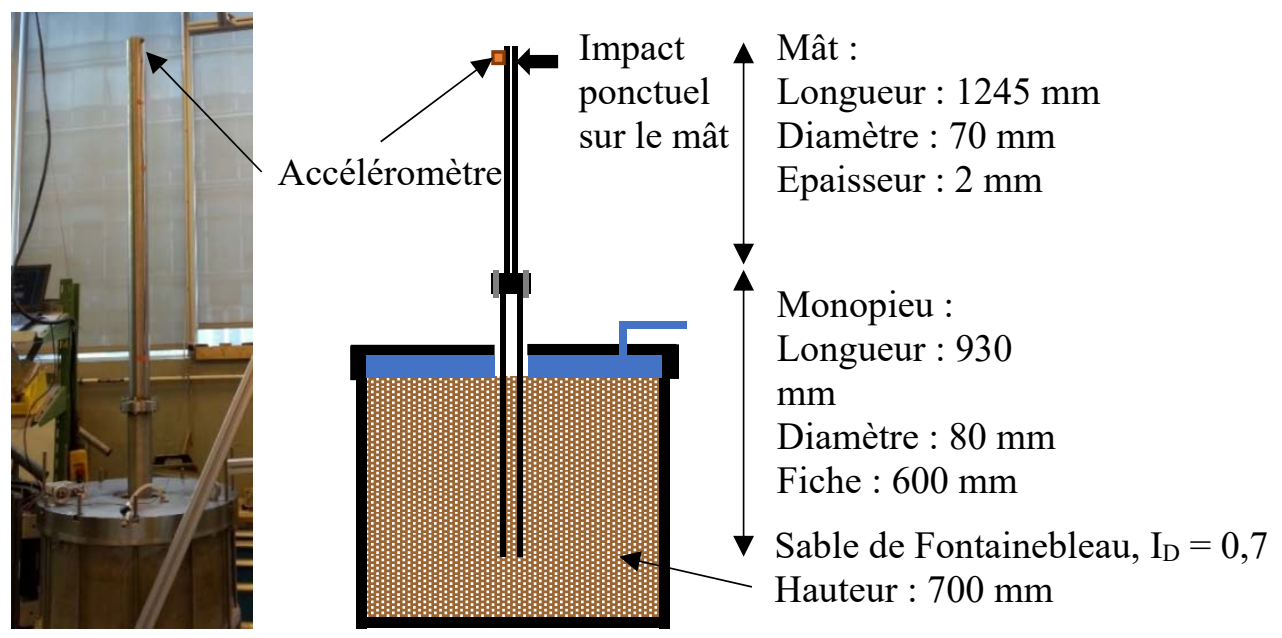

Figure 3. Photo et schéma du dispositif expérimental pour l'évaluation de la $1^{\text {ère }}$ fréquence propre du modèle réduit.

\subsection{Evaluation à l'aide des méthodes analytiques}

En se basant sur les caractéristiques du modèle réduit, la première fréquence propre de la structure a été évaluée à l'aide des quatre méthodes décrites précédemment. Pour la méthode de référence $M 1$ et la méthode $M 2$, l'influence de la contrainte verticale a été prise en compte dans le calcul en faisant varier en conséquence la profondeur dans le calcul du coefficient de réaction du sol $E_{p y}$. La méthode de la longueur d'encastrement équivalente ne permet pas d'inclure l'impact de $\sigma_{V}^{\prime}$, le calcul a donc été fait respectivement pour une longueur d'encastrement de 3,3D et 3,7D. Enfin, pour la méthode $M 4$, les deux approches ont été retenus pour le calcul de la raideur des ressorts latéral et de torsion: le calcul basé sur l'Eurocode $8\left(M 4_{1}\right)$ et le calcul basé sur la méthode d'ADHIKARI \& BHATTACHARYA (2012) notée M42. La variation de $\sigma_{V}^{\prime}$ a été considérée en faisant varier le module d'Young et le module de cisaillement du sol. Les résultats obtenus pour l'ensemble de ces méthodes sont récapitulés sur la figure 4. 


\section{XVèmes Journées Nationales Génie Côtier - Génie Civil \\ La Rochelle, 29 au 31 mai 2018}

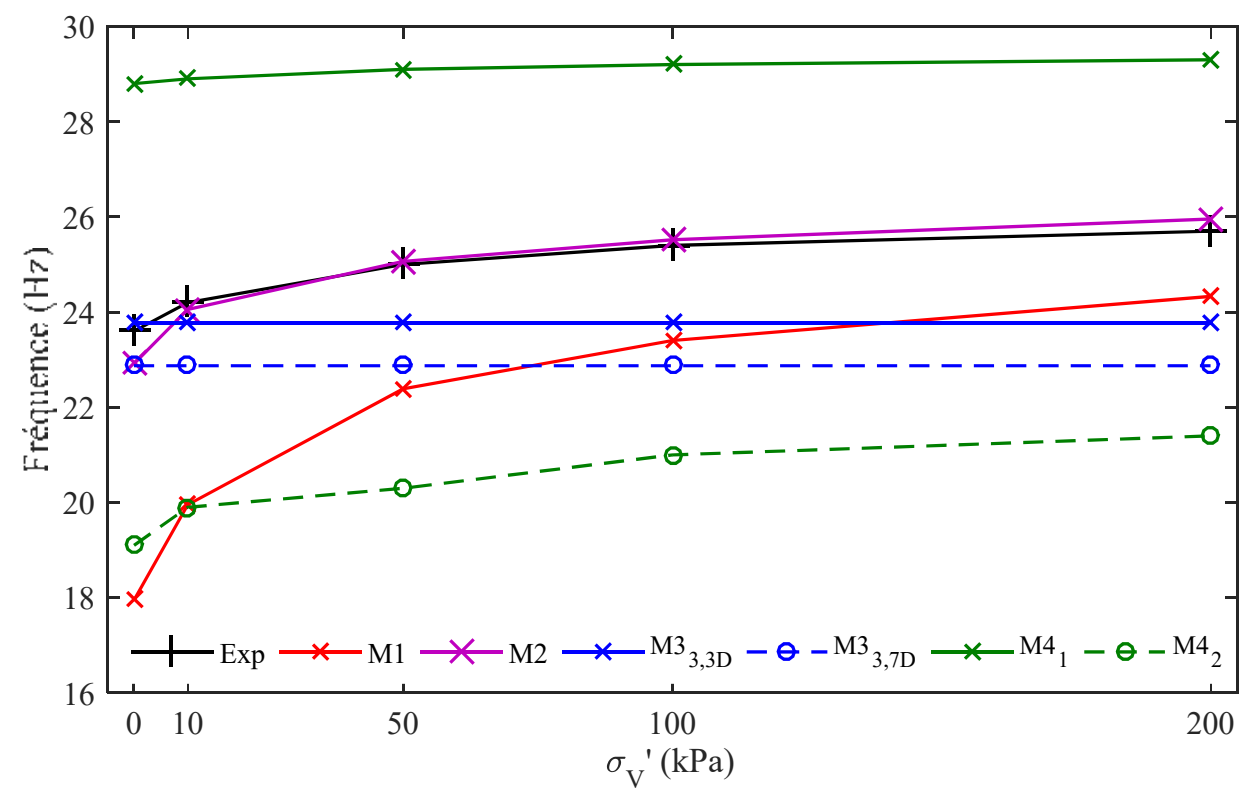

Figure 4. Evolution de la 1ère fréquence propre du modèle réduit en fonction de la contrainte verticale appliquée au massif de sable, résultats expérimentaux (exp), résultats pour les méthodes M1, M2, M3 (pour 3,3D et 3,7D) et M4.

\subsection{Discussion}

Comme souligné précédemment, la méthode de référence $M 1$ sous-estime effectivement l'interaction sol-structure, la première fréquence propre étant sous-estimée avec une erreur de $13 \%$. La méthode $M 2$, donne la meilleure évaluation avec une erreur relative moyenne de $0,4 \%$. La méthode $M 3$ ne permet qu'une évaluation pour un massif de sable à surface libre. Bien que limitée, cette méthode permet d'obtenir facilement et rapidement une première estimation avec une erreur relative comprise entre $1 \%$ (pour $L$ $=3,3 D$ ) et $3 \%$ (pour $L=3,7 D$ ). Enfin, la méthode $M 4$ présente les moins bons résultats avec une forte surestimation (17\%) pour l'approche Eurocode 8 et une forte sousestimation (18\%) pour la méthode d'ADHIKARI \& BHATTACHARYA.

En conclusion, la méthode d'encastrement équivalente semble être une approche adéquate pour obtenir une première évaluation. La méthode de KALLEHAVE et al. (2012) est la méthode la plus appropriée pour un monopieu n'ayant pas un comportement flexible. La méthode développée par ADHIKARI \& BHATTACHARYA (2012) nécessite une évaluation plus fine des raideurs des ressorts qui représentent le facteur majeur dans le calcul de la première fréquence propre.

\section{Conclusion}

Dans cette étude, quatre méthodes d'évaluation de la première fréquence propre d'une éolienne offshore ont été présentées et comparées en se basant sur un modèle réduit à l'échelle 1/60 de l'éolienne NREL 5 MW. 


\section{Thème 5 - Énergies et ressources marines}

La méthode d'encastrement équivalente permet une première évaluation satisfaisante avant d'initier des calculs plus conséquents. Une évaluation plus fine est obtenue à l'aide de la méthode de KALLEHAVE et al. (2012). Ces résultats vont dans le sens de KALLEHAVE et al. (2015) : une prise en compte du diamètre et des caractéristiques du sol dans l'expression des courbes $p$-y permet d'améliorer considérablement l'évaluation de l'interaction sol-structure et donc la première fréquence propre.

\section{Références bibliographiques}

ADHIKARI S., BHATTACHARYA S. (2012). Dynamic analysis of wind turbine towers on flexible foundations. Shock and Vibrations, Vol. 19(1), pp 37-56. https://doi.org/10.3233/SAV-2012-0615

API (2000). Planning, designing and constructing fixed offshore platforms - Working stress design. American Petroleum Institute Recommended Practice 2A-WSD.

DNV (2010). Offshore standard: Design of offshore wind turbine structure. Det Norske Veritas, OS-J101.

JONKMAN J., BUTTERFIELD S., MUSIAL W., SCOTT G. (2009). Definition of a 5MW reference wind turbine for offshore system development. National Renewable Energy Laboratory, NREL/TP-500-38060.

KALLEHAVE D., LIINGARD M.A., LEBLANC C. (2012). Modification of the API p$y$ formulation of initial stiffness of sand. Offshore Site Investigation and Geotechnics: Integrated Technologies - Present and Future, 12 - 14 September, London, UK.

KALLEHAVE D., LEBLANC C., TROYA A. (2015). Observed variations of monopile foundation stiffness, Frontiers in Offshore Geotechnics III, pp 717-722. https://doi.org/10.1201/b18442-98

KUHN M., VAN BUSSEL G.J.W., SCHONTAG C., COCKERILL T.T., HARRISON R., HARLAND L.A., VUGTS J.H. (1998). Methods assisting the design of offshore wind energy conversion systems. Opti OWECS final rep., Vol. 2, Delft: Inst. for Wind Energy. LE T., ARGOUL P. (2004). Continuous wavelet transform for modal identification using free decay response, Journal of Sound and Vibration, Vol. 277, pp 73-100. https://doi.org/10.1016/j.jsv.2003.08.049

MURCHISON J., O'NEIL M. (1984). Evaluation of $p-y$ relationships in cohesionless soils. Analysis and Design of Pile Foundations, Proceedings of a Symposium in conjunction with the ASCE National Convention, pp 174-191.

SØRENSEN S.P.H., IBSEN L.B., AUGUSTESEN A.H. (2010). Effects of diameter on initial stiffness of $p$-y curves for large-diameter piles in sand. $7^{\text {th }}$ European Conference on Numerical Methods in Geotechnical Engineering, Trondheim, Norway, 2-4 June 2010, C R C Press LLC, London, pp 907-912.

THIEKEN K., ACHMUS M., LEMKE K. (2015). A new static p-y approach for piles with arbitrary dimensions in sand. Geotechnik, Vol. 38, pp 267-288. https://doi.org/10.1002/gete.201400036 